\title{
Predicting the Spatio-Temporal Evolution of Chronic Diseases in Population with Human Mobility Data
}

\author{
Yingzi Wang ${ }^{1,2 *}$, Xiao Zhou ${ }^{2}$, Anastasios Noulas ${ }^{3}$, Cecilia Mascolo ${ }^{2}$, Xing $\mathbf{X i e}^{1}$, Enhong Chen ${ }^{1}$ \\ ${ }^{1}$ University of Science and Technology of China \\ ${ }^{2}$ University of Cambridge \\ ${ }^{3}$ Center for Data Science of New York University \\ yingzi@mail.ustc.edu.cn, xz331@cam.ac.uk,noulas@nyu.edu, \\ cm542@cam.ac.uk, xing.xie@gmail.com, cheneh@ustc.edu.cn
}

\begin{abstract}
Chronic diseases like cancer and diabetes are major threats to human life. Understanding the distribution and progression of chronic diseases of a population is important in assisting the allocation of medical resources as well as the design of policies in preemptive healthcare. Traditional methods to obtain large scale indicators on population health, e.g., surveys and statistical analysis, can be costly and time-consuming and often lead to a coarse spatio-temporal picture. In this paper, we leverage a dataset describing the human mobility patterns of citizens in a large metropolitan area. By viewing local human lifestyles we predict the evolution rate of several chronic diseases at the level of a city neighborhood. We apply the combination of a collaborative topic modeling (CTM) and a Gaussian mixture method (GMM) to tackle the data sparsity challenge and achieve robust predictions on health conditions simultaneously. Our method enables the analysis and prediction of disease rate evolution at fine spatio-temporal scales and demonstrates the potential of incorporating datasets from mobile web sources to improve population health monitoring. Evaluations using real-world check-in and chronic disease morbidity datasets in the city of London show that the proposed CTM+GMM model outperforms various baseline methods.
\end{abstract}

\section{Introduction}

Recent studies show that many chronic and malignant diseases, e.g., heart disease, diabetes, and cancer, are extremely prevalent in our society [Siegel et al., 2015]. To estimate disease penetration in a population and to be then able to implement appropriate health-care and preventative measures, healthcare administrators often perform statistical analysis from the records of hospital visits or conduct survey among a sample of residents. The analysis and survey usually incur

\footnotetext{
*This work was done while Yingzi Wang was a visitor at the University of Cambridge
}

high labor costs and are time-consuming. In addition, they often lead to coarse estimates both spatially and temporally.

Although chronic diseases are, to some extent, related to patients' genetics, recent studies have shown that $70 \%$ to $90 \%$ of chronic diseases can be attributed to other factors [Rappaport and Smith, 2010]. For example, there is a causal relationship between chronic diseases and our daily habits, such as diet [McCullough et al., 2002] and alcohol consumption [Martínez, 2005], which reflect different aspects of humans' lifestyles. Lifestyle depicts typical routine lives of people. Large-scale human mobility data collected through location-based social network services can act as a proxy for human lifestyle [Yuan et al., 2013]. For instance, regular visits to college libraries, gyms, and lecture theaters, may correspond to the lifestyle of a college student, while a record of constant visits to meeting rooms and restaurants may indicate the lifestyle of a white-collar employee. These lifestyles are correlated with, and may reveal, certain chronic disease conditions of populations.

The connection between human mobility and diseases has been explored before. Several previous studies have investigated the outbreaks of infectious diseases via social ties and human mobility patterns [Meyers, 2007]. For chronic diseases, researchers have explored the association between human online activities and obesity [Mejova et al., 2015]. However, little attention has been paid to lifestyes as a bridge between human mobility and chronic diseases nor exploring such correlation together with diseases-location similarity to predict residents' health.

In this work, we leverage the correlations between health and lifestyle reflected in human mobility to predict human health progression in populations. Using such data for these estimates gives unprecedented spatial and temporal granularity to the analysis but has also the potential to lower the costs of these studies and enable them to be applicable to regions for which other techniques would be deemed impractical or too expensive (e.g. developing regions). Here, we use human visitation patterns (check-ins) estracted from Foursquare (a location intelligence application) and the statistics of chronic disease morbidity in the London metropolitan area (presented on the government opening data website of the UK). We capture regional lifestyles as reflected in Foursquare mobility 
data, and apply a hybrid model to improve the prediction of public health conditions over simply using historic dataset. In summary, this paper offers the following contributions:

- We explore the correlations between human mobility patterns and health conditions and apply a method which combines Gaussian mixture models (GMM) with collaborative topic modeling (CTM) to predict the health levels of a population, i.e., leveraging "where they go" to help predict "how healthy they are".

- We get clues about human lifestyles from mobility patterns of residents, assuming that the groups of visited POIs are proxies for different lifestyles. We then exploit these inputs to identify fine-grained spatio-temporal associations between these lifestyles and chronic diseases for local populations.

- We collect real-world chronic disease and check-in data to evaluate our method and analyse the correlation between lifestyles and chronic diseases. Compared with methods using historic information solely, the proposed method shows a $45.7 \%$ reduction in mean square erro (MSE) and a 1.67 times increase in R-squared value $\left(R^{2}\right)$.

\section{Related Work}

Disease prediction. Many existing studies have tried to understand the spread of infectious diseases and forecast their outbreaks. Some works have analysed the social or contact networks formed by connections among individuals and human mobility patterns to model the outbreaks of infectious diseases [Meyers, 2007], while some others utilise large amounts of users' status posts on social networks, such as Twitter, to analyse the public health on a large scale [Paul and Dredze, 2011]. Some other studies target on chronic diseases. Matic et al. [Matic and Oliver, 2016] seek help from smartphone-based health applications and wearable devices to continuously record human behaviour to analyse mentalhealth condition of individuals. The work [Mejova et al., 2015] employed both Foursquare and Instagram data to assess the relationship between fast food and obesity. Mason et al. [Mason et al., 2018] found that people who lived far away from fast-food resturants were more likely to have small waist circumference, espetially for women. Howere, there is a noticeable lack of research about the effects of human mobility patterns on the development of chronic diseases in small urban areas. To the best of our knowledge, no one has explored the similarities between chronic diseases and urban regions simultaneously. Our work aims to fill this gap.

Human mobility analysis. Patterns of human mobility are predictable and reflect how the residents of a certain area live in the physical world [Cho et al., 2011]. Many scholars have tried to learn such patterns in order to predict the movement of individuals [Gao et al., 2012]. Extensive research efforts have also been focused on, e.g., finding typical travel sequences by studying users' check-in trajectories [Zheng et al., 2009], predicting users' moving patterns by exploiting both the regularity of human mobility and influence of others [Wang et al., 2015], and making location recommendations with graphical models that integrates users' preferences with their sequential movement patterns [Wang et al., 2016]. Different from above studies, in this paper we investigate the correlations between residents' chronic disease development and their lifestyles indicated by their frequently visited venues and mobility habits.

Topic and Gaussian mixture models. Our analysis utilises topic modeling [Blei et al., 2003] and Gaussian mixture models [Friedman and Russell, 1997]. Topic modeling has been widely used generate latent topics of documents [Wang and Blei, 2011] and learn human habits in daily lives [Yuan et al., 2013]. Gaussian mixture models are often used to describe the joint effect of multiple segments and factors [Bilmes and others, 1998]. It is widely utilised on cluster problems because of its unconstrained covariance structure and flexible application scenarios.

\section{Disease Rate Evolution Prediction}

\subsection{Problem Definition}

Human mobility records, e.g., the check-ins, reflect people's movements in the physical world and to some extent reveal their lifestyles, which gradually affect their health conditions. Here we utilise the check-in dataset of Foursquare and the chronic disease dataset in London as an example for analysis. The Foursquare dataset contains check-in records from Dec. 2010 to Dec. 2013 created at 18,018 POI venues in 426 categories, e.g., fast food restaurant, gym, park, etc. There are over 4 million check-in transition between pairs of POIs, in each of which we have check-in timestamp and venue id (no user information). The chronic disease dataset contains the morbidity of 20 chronic diseases of 567 wards in London.

To exam the relationship between chronic diseases and human mobility patterns extracted from location-based services, we employ Pearson correlation analysis. More specifically, the correlation results between evolution rate of 7 most common chronic diseases and check-in amount of 17 categories of POIs from 2010 to 2013 in London are tested and presented in Figure 1(a). Here, for each chronic disease, we sort the disease evolution rates of 567 wards and split the ranking list into $r$ segments averagely (we set $r=19$, so there are 30 wards in each of the first 18 segments and 27 wards in the last one). We calculate the mean value of disease evolution rate and mean check-in volume of each category of POI in every segment to get two $r$-length sequences. Then, through calculating the correlation coeficient between the two types of sequences, we find that several categories, such as Malaysian restaurants, Chinese restaurants, and fast food restaurants, have high positive correlations with most of the 7 diseases except cancer. Some diseases have similar correlations with all the 17 categories of POIs, e.g. hypertension, heart failure, and obesity.

There are many confounding factors influencing population health across geographies. Our goal is not to draw causal conclusions regarding health. Instead we mine georeferenced data emerging in urban environments so as to inform prediction models and attain better prediction results. Urban activities of users engaging with location intelligence systems can be indicative of lifestyle choices, some of which could be linked to health, yet describing a causal link is not the purpose of this paper.

People's health conditions can be treated as dynamic factors. A healthy lifestyle may not prevent illness but may re- 


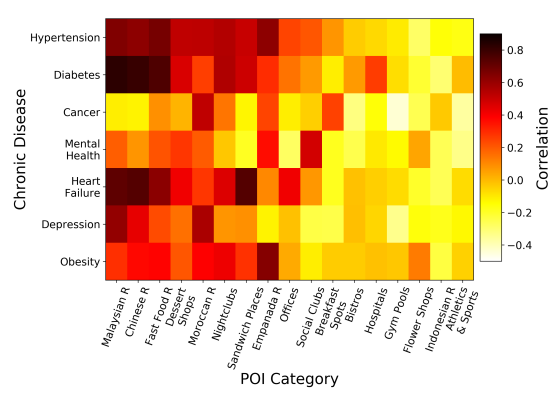

(a)

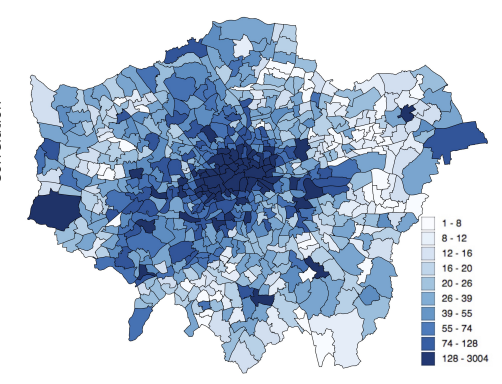

(b)

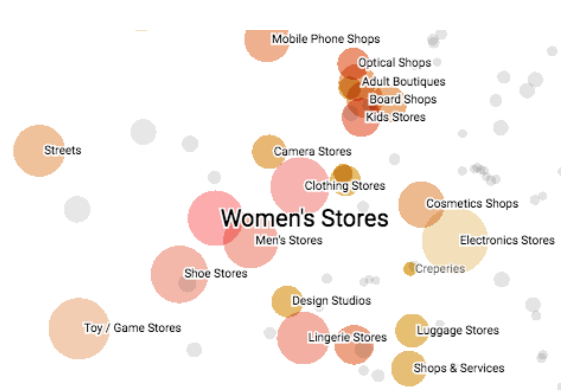

(c)

Figure 1: (a) Correlation between the evolution rate of 7 chronic diseases and check-in amount of 17 POI categories. (b) Distribution of the amount of POIs in the 630 wards of London. (c) 3-D projection of embedding results for POI categories.

duce the risk. Therefore, we focus on how people's lifestyles may influence the development of these chronic diseases. Assume there are $W$ regions in an area, represented as $\mathcal{R}=$ $\left\{r_{1}, r_{2}, \ldots, r_{W}\right\}$. Let $\mathcal{D}=\left\{d_{1}, d_{2}, \ldots, d_{S}\right\}$ denote $S$ chronic diseases and $\boldsymbol{H} \in R^{W \times S}$ denote the evolution matrix of all the $S$ diseases in $W$ regions, where $h_{w, s}$ is the evolution rate of disease $d_{s}$ from last year to this year in region $r_{w}$ (a positive number denotes an increasing rate and a negative number denotes an decreasing rate). In practical terms, the disease data for some regions might be unavailable due to a tight budget. If we can only collect data in a subset $\widehat{R}$ regions of $R$, which fill in $\widehat{W}$ rows in the region-disease matrix $\boldsymbol{H}$ and let others be zero (we denote $\boldsymbol{H}$ matrix with zero rows by $\widehat{\boldsymbol{H}}$ ), our goal is to predict the chronic disease development of the rest $\widetilde{W}=W-\widehat{W}$ regions in set $\widetilde{\mathcal{R}}$.

\subsection{Overview}

We adapt a prediction method which systematically integrates collaborative topic modeling and Gaussian mixture approach. Specifically, we firstly leverage an embedding method and a Gaussian mixture approach to aggregate categories of venues into several clusters according to their check-in patterns. Through that, we obtain a denser region-cluster-of-venues matrix. Then we apply a collaborative topic modeling method to extract lifestyle patterns of each region from human's check-in mobility. We hypothesise that lifestyle patterns in all regions and chronic disease evolution rates in some of the regions can be alble to help exploring the chronic disease conditions in the missing regions.

\subsection{Method}

Venue Aggregation. Assuming there are $N$ categories of venues, denoted as $\mathcal{V}=\left\{v_{1}, v_{2}, \ldots v_{N}\right\}$, we can collect the check-in amount of each category in $\mathcal{V}$ and each region in $\mathcal{R}$ (mentioned in Section 3.1), and build a region-category matrix $\boldsymbol{Y} \in R^{W \times N}$. However, the spatial distribution of POIs is usually unbalanced in the city. In Figure 1(b), the amounts of Foursquare POIs in the 630 wards are presented. We can clearly see that central London and the region containing Heathrow Airport (the deep blue region on the left) have denser POI distribution. However, in some other regions the amount of POIs are considerably sparse, which leads to the sparsity of matrix $\boldsymbol{Y}$. To address this problem, we extract users' similar check-in preferences for some categories of
POIs and aggregate these categories into a cluster. For example, if users often go shopping after having French or Italian food, we will cluster French and Italian food into a group.

To aggregate venues based on check-in patterns, we firstly represent each category of POI as a feature vector. Those categories with similar check-in patterns will have smaller vector distances from each other. This is similar to the embedding task in natural language processing, where each category of POI can be seen as a word, and users' transitions between categories of POIs is analogous to a sentence. Here we embed each category of POI into a $P$-length vector through word2vec method [Rehurek and Sojka, 2010]. Figure 1(c) shows a part of the 3-D projection ${ }^{1}$ of embedding result for all the 426 categories of POIs when $P$ is set to 100 . Each circle denotes one category. The colored circles are the categories having shortest cosine distances to the category "women's stores". Redder colors represent closer relationships. The size of a circle indicates it's "depth" from the surface of screen (foreshortening effects). We can observe that shoe stores, clothing stores, lingerie stores, and men's stores, have the most similar check-in patterns with women's stores.

We aggregate the $N$ categories of POIs into $C$ clusters by adopting Gaussian mixture method (GMM) [Friedman and Russell, 1997]. Compared with other cluster methods, e.g., k-means, GMM provides unconstrained covariance structure for each cluster, making the method more flexible. As illustrated in Figure 2, $\phi \in R^{N \times P}$ is the venue-embedding matrix. The GMM can be described as follows:

$$
\begin{gathered}
\boldsymbol{\pi}_{n} \sim \operatorname{Dirichlet}(\gamma), \\
\boldsymbol{\sigma}_{c} \sim \Gamma\left(\tau, \sigma_{0}\right), \quad \boldsymbol{\mu}_{c} \sim \mathcal{N}\left(\mu_{0}, \nu \boldsymbol{\sigma}_{c}\right), c=1, \ldots, C, \\
g_{n} \sim \operatorname{Categorical}\left(\boldsymbol{\pi}_{n}\right), \quad \boldsymbol{\phi}_{n} \sim \mathcal{N}\left(\boldsymbol{\mu}_{g_{n}}, \boldsymbol{\sigma}_{g_{n}}\right), n=1, \ldots, N,
\end{gathered}
$$

where $\boldsymbol{\pi}_{n}$ and $g_{n}$ are the parameter of categorical distribution and the component of the $n^{t h}$ observation respectively. $\boldsymbol{\mu}_{c}, \boldsymbol{\sigma}_{c}$ are the parameters of Gaussian distribution of component $c$. $\gamma, \tau, \sigma_{0}, \mu_{0}, \nu$ are the shared hyperparameters. Let $\boldsymbol{X} \in R^{W \times C}$ represent the region-cluster matrix, which can be estimated through parameter $\boldsymbol{\pi}$ and check-in matrix $\boldsymbol{Y}$ :

$$
\boldsymbol{X}=\boldsymbol{Y} \cdot \boldsymbol{\pi},
$$

where $x_{i, j}$ denotes the check-in amount of venue cluster $j$ in region $r_{i}$. Figure 2 presents the association from $\boldsymbol{Y}$ and $\boldsymbol{\pi}$ to matrix $\boldsymbol{X}$ through dotted lines.

\footnotetext{
${ }^{1}$ http://projector.tensorflow.org/
} 
Collaborative Topic Model. Until now, we have obtained the region-cluster matrix $\boldsymbol{X}$. Our objective is to extract lifestyle information from $\boldsymbol{X}$ helping to predict chronic disease conditions in missing regions. Traditional probabilistic matrix factorization (PMF) is a perfect method for recommendation tasks, leveraging the similarity among different users and items to complement the user-item matrix [Salakhutdinov and Mnih, 2007]. Similarly, as presented in Figure 1(a), similarities exit among diseases when analysing the correlation between diseases and check-ins. If we assume that similarities could also be found among regions, we can in the same way factorize regions' disease evolution rate matrix $\boldsymbol{H}$ into two low dimensional latent matrices $\boldsymbol{L}$ and $\boldsymbol{\Lambda}$, both with dimension $K$. We denote $K$ as the number of latent lifestyles, vector $\boldsymbol{L}_{i}$ as the weight of latent lifestyles in region $r_{i}$, and vector $\boldsymbol{\Lambda}_{j}$ as the influence from latent lifestyles on chronic disease $d_{j}$. Thus we can generate $\boldsymbol{H}$ through the distribution:

$$
h_{i, j} \sim \mathcal{N}\left(\boldsymbol{L}_{i} \cdot \boldsymbol{\Lambda}_{j}^{\top}, \varsigma_{i, j} \lambda_{H}^{2}\right),
$$

where $\varsigma_{i, j}$ is 0 if the data of $h_{i, j}$ is missing, and 1 otherwise. The distribution of region and disease vectors are:

$$
\boldsymbol{L}_{i} \sim \mathcal{N}\left(0, \lambda_{L}^{2} \boldsymbol{I}_{K}\right), \quad \boldsymbol{\Lambda}_{j} \sim \mathcal{N}\left(0, \lambda_{\Lambda}^{2} \boldsymbol{I}_{K}\right),
$$

where $\boldsymbol{I}_{K}$ is a $K$-dimensional identity matrix. A common way to optimize parameters $\boldsymbol{L}$ and $\boldsymbol{\Lambda}$ is to minimize the squarederrors objective function with regularization terms:

$$
\Omega=\boldsymbol{I} \odot\left\|\boldsymbol{H}-\boldsymbol{L} \boldsymbol{\Lambda}^{\top}\right\|_{F}^{2}+\frac{\lambda_{H}^{2}}{\lambda_{L}^{2}}\|\boldsymbol{L}\|_{F}^{2}+\frac{\lambda_{H}^{2}}{\lambda_{\Lambda}^{2}}\|\boldsymbol{\Lambda}\|_{F}^{2},
$$

where $\|\cdot\|_{F}$ denotes the Frobenius norm and $\odot$ denotes the Hadamard product operator [Kolda and Bader, 2009].

However, as only the data of $\widehat{R}$ regions are avaliable, information for leveraging similarities among the missing rows and the existing rows in matrix $\widehat{\boldsymbol{H}}$ is lacking. Hence we use the region-cluster matrix $\boldsymbol{X}$ obtained in the last section. We assume each region is characterized by a particular set of lifestyles, and each cluster of categories of POIs may reflect human's various lifestyles in different probabilities. It is similar to a topic structure: if we regard all the regions as documents, the check-in patterns of various POI clusters in a region can be considered as the words in a document. In analogy with the assumption that each topic is described by several representative words, each lifestyle is reflected in different check-in patterns. Intuitively, a typical topic model, LDA [Blei et al., 2003], can be applied here to model the lifestyles in different regions.

However, LDA does not tackle the main problem in this work: how to leverage the lifestyles extracted from checkin mobility to fill the missing parts in matrix $\widehat{\boldsymbol{H}}$. We adopt the collaborative topic model (CTM) proposed in [Wang and Blei, 2011] here to combine probabilistic matrix factorization and topic modeling. Different from the assumption in [Wang and Blei, 2011], that offsets exist between the document-topic factor factorized from document-user part and document-word part respectively, we propose that people's general lifestyles, reflecting in check-in patterns and health conditions, are relatively consistent. POIs' visiting and chronic disease condition are just two views of lifestyles,

\begin{tabular}{|c|c|c|}
\hline asthma & \multirow{2}{*}{$\begin{array}{c}\text { chronic obstructive } \\
\text { pulmonary } \\
\text { disease (COPD) }\end{array}$} & heart failure \\
\hline $\begin{array}{c}\text { atrial } \\
\text { fibrillation }(\mathrm{AF})\end{array}$ & & $\begin{array}{c}\text { learning } \\
\text { disabilities (LD) }\end{array}$ \\
\hline cancer & diabetes & mental health \\
\hline \multirow{2}{*}{$\begin{array}{l}\text { chronic kidney } \\
\text { disease (CKD) }\end{array}$} & epilepsy & obesity \\
\hline & hypertension & palliative care \\
\hline \multirow{2}{*}{$\begin{array}{l}\text { coronary heart } \\
\text { disease (CHD) }\end{array}$} & hypothyroidism & smoking \\
\hline & \multirow{3}{*}{$\begin{array}{l}\text { heart failure } \\
\text { due to LVD } \\
\text { (HFLVD) }\end{array}$} & \multirow{3}{*}{$\begin{array}{l}\text { stroke or transient } \\
\text { ischaemic attacks } \\
\text { (stroke or TIA) }\end{array}$} \\
\hline dementia & & \\
\hline depression & & \\
\hline
\end{tabular}

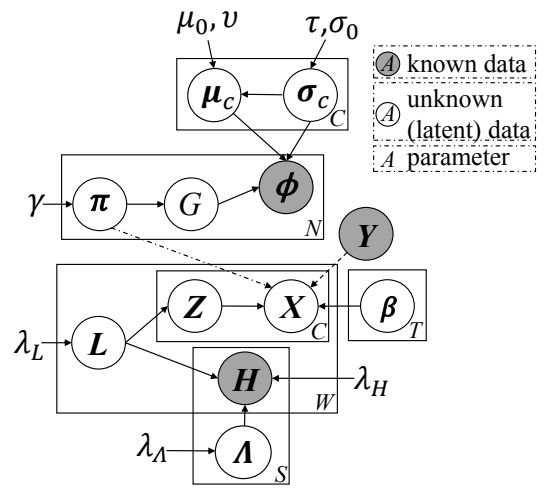

Figure 2: Illustration of CTM+GMM method.

Table 1: 20 chronic diseases and their abbreviations.

where the former one is the perspective in people's daily life activities, and the latter one is how these lifestyles influence people's health status. Therefore, we leverage the same factor $\boldsymbol{L}$, to represent region-lifestyle interactions in topic modeling. Specifically, the generative process of the hybrid method is as follows (illustrated in the bottom part of Figure 2):

1. For each chronic disease $j$, draw disease latent factor $\boldsymbol{\Lambda}_{j} \sim \mathcal{N}\left(0, \lambda_{\Lambda}^{2} \boldsymbol{I}\right)$.

2. For each region $i$ :

(a) Draw lifestyle factor $\boldsymbol{L}_{i} \sim \operatorname{Dirichlet}\left(\lambda_{L}\right)$.

(b) For each category of venues $x_{i, c}$ :

(i) Choose lifestyle assignment $z_{i, c} \sim \operatorname{Mult}\left(\boldsymbol{L}_{i}\right)$.

(ii) Choose a category of venues $x_{i, c} \sim \operatorname{Mult}\left(\boldsymbol{\beta}_{z_{i, c}}\right)$.

3. For each region-disease pair $h_{i, j}$, draw $h_{i, j} \sim \mathcal{N}\left(\boldsymbol{L}_{i}\right.$. $\left.\boldsymbol{\Lambda}_{j}^{\top}, i_{i, j} \lambda_{H}^{2}\right)$,

where $\boldsymbol{\beta}_{z_{t}}$ is the distribution of POI clusters in lifestyle $z_{t}$.

Optimization. We apply EM method [Bilmes and others, 1998] to estimate the parameters $\left\{\boldsymbol{\mu}_{c}, \boldsymbol{\sigma}_{c}^{2}\right\}(c=1, \ldots, C)$ and $\boldsymbol{\pi}$ in GMM part. For the CTM part, we need to estimate the parameters $\{\boldsymbol{L}, \boldsymbol{\Lambda}\}$, where factor $\boldsymbol{L}$ is employed both in PMF and topic modeling. We iteratively optimize parameters by two steps. Firstly, for topic modeling, we estimate $\boldsymbol{L}$ through EM method, which is a typical optimization method for topic modeling. Then in the second step, we apply Gradient Descent method to estimate $\{\boldsymbol{L}, \boldsymbol{\Lambda}\}$ (we use the $\boldsymbol{L}$ factor in the first step as the initialization here). We then go back to step 1 and set $\boldsymbol{L}$ as the prior of region lifestyle distribution.

\section{Evaluation}

\subsection{Set-Up}

Data. We use three datasets in our experiment: 


\begin{tabular}{|l|l|}
\hline Cluster 1 & Cluster 2 \\
\hline \hline gardens, Russian r, malls, & hotels, jazz clubs, skate parks, \\
office supplies stores, banks, & tattoo parlors, nightlife spots, \\
art galleries, music stores, & Latin American r, social clubs, \\
bowling alleys, theme park, & cupcake shops, smoke shops, \\
dessert shops, comedy clubs, & beer stores, laundry services, \\
Taiwanese r, college libraries, & dinners, modern European r, \\
bookstores, souvenir shops, & convenience stores, bridges \\
snack places, cosmetics shops & \\
\hline
\end{tabular}

Table 2: Categories of POIs in 2 clusters (r: restaurants).

Foursquare dataset: The check-in records from Dec. 2010 to Dec. 2013 and the POI information in London. The details of Foursquare dataset are introduced in Section 3.1. In August 2015, Foursquare had more than 50 million active users and more than 10 billion check-ins already. The dataset used in this research is shared directly by Foursquare under a research contract agreement.

Boundary-line dataset of London: The dataset is collected from UK government websites ${ }^{2}$, which contain the shapefiles of ward-level (electoral districts at sub-national level) boundary lines in London. In total, there are 630 wards. This spatial data contains the shape line, name, and id of each ward (shape lines are shown in Figure 1(b)).

Disease dataset: We collect the data from a government open data website of $\mathrm{UK}^{3}$. They publish the population, the annual morbidity (patients/population in an area) of 19 prevalence diseases (Table1), and the utilization rate of "palliative care" from year 2005 to 2015 . Since we consider palliative care ${ }^{4}$ as an indicator for the morbidity of malignant diseases (it is a specialized medical care for people with lifethreatening illness), we call it "disease" here. The data for each year is from Apr. of one year to Mar. of the next year. For convenience, we use dataset "2013", for example, to represent the annual dataset from Apr. 2013 to Mar. 2014 in the rest of the paper. We collected the data from 2009 to 2013, consistent with the period of check-in data. The dataset contains the popularity and morbidity data of 567 wards in London (no data for the rest 63 wards).

Metrics. We apply two metrics here to evaluate the prediction performance: (MSE) and $R^{2}$ score:

$$
\begin{gathered}
M S E\left(\boldsymbol{H}, \boldsymbol{H}^{\prime}\right)_{\widetilde{\mathcal{R}}}=\frac{1}{\widetilde{W} \cdot S} \sum_{r_{i} \in \widetilde{\mathcal{R}}} \sum_{j=1}^{S}\left(h_{r_{i}, j}-h_{r_{i}, j}^{\prime}\right)^{2}, \\
R^{2}\left(\boldsymbol{H}, \boldsymbol{H}^{\prime}\right)_{\widetilde{\mathcal{R}}}=\frac{1}{\widetilde{W}} \sum_{r_{i} \in \widetilde{\mathcal{R}}}\left(1-\frac{\sum_{j=1}^{S}\left(h_{r_{i}, j}-h_{r_{i}, j}^{\prime}\right)^{2}}{\sum_{j=1}^{S}\left(h_{r_{i}, j}-\bar{h}_{r_{i}}\right)^{2}}\right),
\end{gathered}
$$

where $h_{r_{i}, j}^{\prime}$ is the prediction result of item $h_{r_{i}, j}$ and $\bar{h}_{r_{i}}=$ $\frac{1}{S} \sum_{j=1}^{S} h_{r_{i}, j} . \widetilde{W}$ is the amount of regions in testing set. $R^{2}$ score reflects how well the model performs in the prediction, considering the error and the mean of true values simultaneously. Here lower $M S E$ and higher $R^{2}$ represent better result.

Baselines. We compare the hybrid method with 4 methods:

\footnotetext{
${ }^{2}$ https://www.ordnancesurvey.co.uk/opendatadownload/products .html\#BDLINE

${ }^{3}$ https://data.gov.uk/dataset/quality_and_outcomes_framework_ac hievement_prevalence_and_exceptions_data

${ }^{4}$ https://en.wikipedia.org/wiki/Palliative_care
}

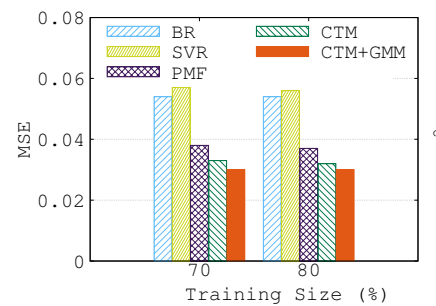

(a) $M S E$

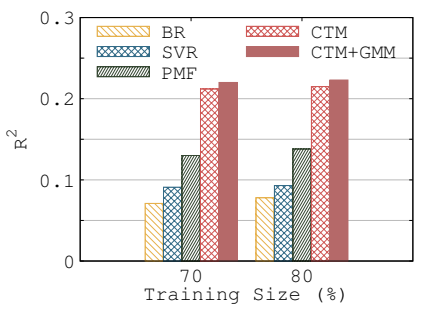

(b) $R^{2}$
Figure 3: Prediction performance of all the evaluated methods on (a) $M S E$ and (b) $R^{2}$, when training size is $70 \%$ or $80 \%$.

- Regression: We apply two regression methods using history data solely: boosting regression (BR) and support vector regression (SVR). They utilise the data of chronic disease evolution rate $(2009$ - 2012) to predict the evolution rate from 2012 to 2013

- Probabilistic matrix factorization (PMF): In PMF, the region-disease matrix $\widehat{\boldsymbol{H}}$ (with missing rows) is decomposed through equation 3 ( $\boldsymbol{H}$ is replaced with $\widehat{\boldsymbol{H}}$ ).

- Collaborative topic modeling (CTM): We compare our method with CTM to emphasize the improvement achieved through venue aggregation.

\subsection{Results}

We compare the prediction performance of our method with baseline models in this section. Here we embed each POI category into a 100-length vector, and produce matrix $\phi \in R^{426 \times 100}$. Each vector represents POI category's features in check-in pattern space, where a shorter distance between two categories indicates more similar check-in patterns. We aggregate the 426 embedded vectors into $C$ clusters through a GMM method. Table 2 shows POI categories in two typical clusters when $C=20$. We can see that most POIs in the first cluster are more about nature (parks, gardens, etc.), art (art galleries, music stores, comedy clubs, etc), and reading (libraries and bookstores), while the second cluster mainly includes nightlife-related venues. The clustering results provide us new perspectives on London citizens' visitation to POIs, which are not totally consistent with our experience. For instance, it is unexpected that bridges have similar checkin patterns with some clubs and nightlife spots. This may be because that the fantastic lighting systems of some famous bridges in London, such as Albert Bridge and Tower Bridge, attract a large number of viewers during the night hours.

Next, we predict the missing rows in matrix $\boldsymbol{H}$, in which item $h_{i, j}$ is the evolution rate of disease $d_{j}$ in ward $r_{i}$ from year 2012 to 2013. $h_{i, j}$ is generated from the equation $h_{i, j}=\left(m_{i, j}^{2013}-m_{i, j}^{2012}\right) / m_{i, j}^{2012}$, where $m$ represents morbidity. Firstly, we obtain the region-cluster matrix $\boldsymbol{X}$ through Equation 1. Then, the latent lifestyle factor $\boldsymbol{L}$ and latent chronic disease factor $\boldsymbol{\Lambda}$ are estimated through CTM in Section 3.3 . Finally, we predict the missing regions $\widetilde{\mathcal{R}}$ in matrix $\boldsymbol{H}$ through Equation 2. We randomly select $70 \%$ and $80 \%$ rows in matrix $\boldsymbol{H}$ as training data and evaluate the prediction performance in the rest rows. Due to the space limit, for all the methods, we tune and select the parameters through training set and show the prediction results on testing set. For 
Proceedings of the Twenty-Seventh International Joint Conference on Artificial Intelligence (IJCAI-18)

\begin{tabular}{|l|l|l|l|l|l|}
\hline Disease & ER & $\mathbf{R}^{2} \mathbf{S}$ & Disease & ER & $\mathbf{R}^{2} \mathbf{S}$ \\
\hline asthma & -0.03 & 0.155 & hypertension & 0.07 & 0.156 \\
AF & 3.17 & 0.166 & hypothyroidism & 2.94 & 0.129 \\
cancer & 7.12 & 0.150 & heart failure & 0.59 & 0.160 \\
CKD & -2.12 & 0.157 & HFLVD & -44.27 & 0.150 \\
CHD & -1.17 & 0.159 & LD & 4.36 & 0.175 \\
COPD & 2.94 & 0.166 & mental health & 2.37 & 0.170 \\
dementia & 11.13 & 0.163 & obesity & -16.10 & 0.133 \\
depression & 12.15 & 0.144 & palliative care & 21.92 & 0.140 \\
diabetes & 3.10 & 0.141 & smoking & 1.18 & 0.181 \\
epilepsy & 2.02 & 0.168 & stroke or TIA & 1.11 & 0.142 \\
\hline
\end{tabular}

Table 3: Average evolution rates of the 20 diseases and their $R^{2}$ scores in prediction (ER: evolution rate (\%), $\mathbf{S}$ : score).

each method, we run the analysis 20 times and show the average results in Figure 3. The latent dimension length $K$ and the amount of lifestyles $T$ (CTM part) are set to 50. $C$, the amount of component (GMM part), is set to 20. It is obvious that CTM+GMM has the best performance on the two metrics. The two regression methods have the highest MSE results and lowest $R^{2}$ scores. We can infer that the historic evolutions of these chronic diseases cannot provide enough regular patterns (e.g. linear or periodic patterns) for future prediction. Compared with traditional PMF, CTM has an average improvement of $13 \%$ in $M S E$ and $59 \%$ in $R^{2}$ score. Moreover, when we add the GMM part to CTM, it furtherly achieves a $7.6 \%$ improvement in $M S E$ and a $3.7 \%$ improvement in $R^{2}$ score.

We show the average evolution rate and the $R^{2}$ scores of all the diseases in predictions through CTM+GMM method from 2012 to 2013 in Table 3 (different from the definition in Section 4.1, we here show the $R^{2}$ scores along each disease). The Pearson correlation coefficient of evolution rate list and $R^{2}$ list in the table is 0.11 , which means that there is no obvious correlation between these two factors: the predictability of a disease is neither positively nor negatively related to its evolution rate. From this table, we can see a significant growth of palliative care during that year, indicating that the morbidity of malignant diseases, e.g., cancer, had increased. Also, the morbidity of the psychiatric and mental diseases, e.g. depression, dementia, and mental health, increased dramatically in that period. Actually, as illustrated in [Muliyala and Varghese, 2010], depression and dementia have complex relationships: depression has been both a risk factor and a prodrome of dementia, which is also reflected in the close evolution rates between them. Good news is that the obesity cases in London decreased in the same period, which has been a serious health concern in UK for a long time. Moreover, we observe that obesity has a relatively low $R^{2}$ score in the experiment for both our method and baselines. This may be due to the fact that different from some incurable chronic diseases, e.g., diabetes [Etuk and others, 2010], of which the morbidity is more stable, people may lose weight through various approaches, making the evolution of obesity more difficult to predict.

\section{Lifestyle and Chronic Diseases}

We present the correlation between the 20 chronic diseases and various lifestyles. We leverage the check-in pattern as a projection of lifestyle. Specifically, we use the lifestylecluster factor ( $\boldsymbol{\beta}$ in Section 3.3), and the disease-lifestyle fac-

\begin{tabular}{|l|l|}
\hline Check-in Lifestyles & Correlated Dis \\
\hline \hline government buildings, pubs, sushi r, plazas, & heart failure, \\
candy stores, steakhouses, burger joints, & attacks, asthma, \\
Subways, Chinese r, churches, bookstores, & COPD, \\
fast food r, coffee shops, nightclubs & coronary heart \\
\hline Indian r, convention centers, fast food r, & disease, diabetes, \\
sandwich places, grocery stores, Thai r, & hypertension, \\
dim sum r, hotel bars, bakeries, hostels, & obesity, HFLVD, \\
nightclubs, fried chicken joints & stroke or TIA \\
\hline \hline dessert shops, organic groceries, gay bars, & cancer, smoking \\
middle eastern r, sports bars, cocktail bars, & chronic kidney, \\
whisky bars, nightclubs, offices, boutiques, & depression, \\
Chinese r, hotel bars, hotels, Italian r & disease, obesity, \\
\hline
\end{tabular}

Table 4: Typical check-in lifestyles and the highly-correlated diseases (Dis: Diseases, r: restaurants).

tor $\boldsymbol{\Lambda}$, to uncover the hidden relationship between chronic diseases and POIs. Some of the observed correlations are consistent with the research findings in clinical medicine and physiology, while others provide new insights into some open problems. We list 3 typical check-in lifestyles and their highly correlated diseases in Table 4.

The top two lifestyles are dominated by fast food venues: fast food restaurants, pizza places, fried chicken joints, and Asian food (Chinese and sushi restaurants). These two lifestyles show association with 10 chronic diseases listed on the right. This is coherent with the statement that some diseases, like heart-related diseases, hypertension, obesity, and diabetes, are correlated with diets high in sugar and fat. Additionally, asthma and chronic obstructive pulmonary disease are also highly correlated with these two lifestyles. This is consistent with previous studies in physiology [Rosenkranz et al., 2010] claiming that a high-fat diet may contribute to chronic inflammatory diseases of airways and lungs.

The third lifestyle is alcohol-oriented, where 6 of the 14 categories of POIs are bars or clubs. The induction of alcohol on some diseases, like cancer, depression, have been proved in previous studies [Martínez, 2005]. However, as illustrated in [White et al., 2009], evidence of an association between alcohol consumption and chronic kidney disease is conflicting. Here we draw inspiration from large-scale human mobility data and provide an intuitive perspective on the positive correlation between lifestyles related to alcohol and chronic kidney disease.

\section{Conclusion}

In this paper, we leverage human mobility data to study the evolution of human health conditions. We embed the POIs into vectors according to human transition patterns to capture their semantic meanings. Then we combine Gaussian mixture methods with collaborative topic modeling to predict health conditions, which is able to deal with data sparsity and extract human lifestyles from check-in patterns. Experiments using real-world datasets indicated that CTM+GMM has a significant improvement on prediction tasks compared to baselines.

\section{Acknowledgments}

We would like to thank Foursquare for making the data available to the University of Cambridge for this study. 


\section{References}

[Bilmes and others, 1998] Jeff A Bilmes et al. A gentle tutorial of the em algorithm and its application to parameter estimation for gaussian mixture and hidden markov models. ICSI, 4(510):126, 1998.

[Blei et al., 2003] David M Blei, Andrew Y Ng, and Michael I Jordan. Latent dirichlet allocation. Journal of machine Learning research, 3(Jan):993-1022, 2003.

[Cho et al., 2011] Eunjoon Cho, Seth A. Myers, and Jure Leskovec. Friendship and mobility: User movement in location-based social networks. In SIGKDD, pages 10821090, 2011.

[Etuk and others, 2010] EU Etuk et al. Animals models for studying diabetes mellitus. Agric Biol JN Am, 1(2):130134, 2010.

[Friedman and Russell, 1997] Nir Friedman and Stuart Russell. Image segmentation in video sequences: A probabilistic approach. In Proceedings of the Thirteenth conference on UAI, pages 175-181. Morgan Kaufmann Publishers Inc., 1997.

[Gao et al., 2012] Huiji Gao, Jiliang Tang, and Huan Liu. gscorr: Modeling geo-social correlations for new checkins on location-based social networks. In CIKM, pages 1582-1586, 2012.

[Kolda and Bader, 2009] Tamara G Kolda and Brett W Bader. Tensor decompositions and applications. SIAM review, 51(3):455-500, 2009.

[Martínez, 2005] María Elena Martínez. Primary prevention of colorectal cancer: lifestyle, nutrition, exercise. In Tumor Prevention and Genetics III, pages 177-211. Springer, 2005.

[Mason et al., 2018] Kate E Mason, Neil Pearce, and Steven Cummins. Associations between fast food and physical activity environments and adiposity in mid-life: crosssectional, observational evidence from uk biobank. The Lancet Public Health, 3(1):e24-e33, 2018.

[Matic and Oliver, 2016] Aleksandar Matic and Nuria Oliver. The untapped opportunity of mobile network data for mental health. In Proceedings of the 10th EAI International Conference on Pervasive Computing Technologies for Healthcare, pages 285-288, 2016.

[McCullough et al., 2002] Marjorie L McCullough, Diane Feskanich, Meir J Stampfer, Edward L Giovannucci, Eric B Rimm, Frank B Hu, Donna Spiegelman, David J Hunter, Graham A Colditz, and Walter C Willett. Diet quality and major chronic disease risk in men and women: moving toward improved dietary guidance. The American journal of clinical nutrition, 76(6):1261-1271, 2002.

[Mejova et al., 2015] Yelena Mejova, Hamed Haddadi, Anastasios Noulas, and Ingmar Weber. \# foodporn: Obesity patterns in culinary interactions. In Proceedings of the 5th International Conference on Digital Health 2015, pages 51-58. ACM, 2015.
[Meyers, 2007] Lauren Meyers. Contact network epidemiology: Bond percolation applied to infectious disease prediction and control. Bulletin of the American Mathematical Society, 44(1):63-86, 2007.

[Muliyala and Varghese, 2010] Krishna Prasad Muliyala and Mathew Varghese. The complex relationship between depression and dementia. Annals of Indian Academy of Neurology, 13(Suppl2):S69, 2010.

[Paul and Dredze, 2011] Michael J Paul and Mark Dredze. You are what you tweet: Analyzing twitter for public health. ICWSM, 20:265-272, 2011.

[Rappaport and Smith, 2010] Stephen M Rappaport and Martyn T Smith. Environment and disease risks. Science, 330(6003):460-461, 2010.

[Rehurek and Sojka, 2010] Radim Rehurek and Petr Sojka. Software Framework for Topic Modelling with Large Corpora. In Proceedings of the LREC 2010 Workshop on New Challenges for NLP Frameworks, pages 45-50, May 2010.

[Rosenkranz et al., 2010] Sara K Rosenkranz, Dana K Townsend, Suzanne E Steffens, and Craig A Harms. Effects of a high-fat meal on pulmonary function in healthy subjects. European journal of applied physiology, 109(3):499-506, 2010.

[Salakhutdinov and Mnih, 2007] Ruslan Salakhutdinov and Andriy Mnih. Probabilistic matrix factorization. In Nips, volume 1, pages 2-1, 2007.

[Siegel et al., 2015] Rebecca L Siegel, Kimberly D Miller, and Ahmedin Jemal. Cancer statistics, 2015. CA: a cancer journal for clinicians, 65(1):5-29, 2015.

[Wang and Blei, 2011] Chong Wang and David M Blei. Collaborative topic modeling for recommending scientific articles. In SIGKDD, pages 448-456. ACM, 2011.

[Wang et al., 2015] Yingzi Wang, Nicholas Jing Yuan, Defu Lian, Linli Xu, Xing Xie, Enhong Chen, and Yong Rui. Regularity and conformity: Location prediction using heterogeneous mobility data. In SIGKDD, pages 1275-1284, 2015.

[Wang et al., 2016] Weiqing Wang, Hongzhi Yin, Shazia Wasim Sadiq, Ling Chen, Min Xie, and Xiaofang Zhou. SPORE: A sequential personalized spatial item recommender system. In ICDE, pages 954-965, 2016.

[White et al., 2009] Sarah L White, Kevan R Polkinghorne, Alan Cass, Jonathan E Shaw, Robert C Atkins, and Steven J Chadban. Alcohol consumption and 5-year onset of chronic kidney disease: the ausdiab study. Nephrology Dialysis Transplantation, 24(8):2464-2472, 2009.

[Yuan et al., 2013] Nicholas Jing Yuan, Fuzheng Zhang, Defu Lian, Kai Zheng, Siyu Yu, and Xing Xie. We know how you live: exploring the spectrum of urban lifestyles. In COSN, pages 3-14. ACM, 2013.

[Zheng et al., 2009] Yu Zheng, Lizhu Zhang, Xing Xie, and Wei-Ying Ma. Mining interesting locations and travel sequences from gps trajectories. In $W W W$, pages 791-800, 2009. 